\title{
Effect of Ezetimibe Monotherapy on Low-Density Lipoprotein Cholesterol and on Markers of Cholesterol Synthesis and Absorption in Japanese Patients With Hypercholesterolemia
}

\author{
Akio Ohta ${ }^{a}$,, Hiroyuki Kato ${ }^{a}$, Satoshi Ishii ${ }^{a}$, Yoshio Nagai ${ }^{\text {a }}$, Yasushi Tanaka ${ }^{a}$
}

\begin{abstract}
Background: The aim of this study was to evaluate effect of ezetimibe monotherapy on serum low-density lipoprotein cholesterol (LDL-C) in Japanese patients and to investigate the association between changes of LDL-C and changes of markers for cholesterol synthesis and absorption.

Methods: Seventy-six hypercholesterolemic patients without statin therapy were enrolled and randomized to two groups, which were an ezetimibe group (group $\mathrm{E}, \mathrm{n}=44$ ) and a control group without ezetimibe treatment that received diet therapy alone (group $\mathrm{C}, \mathrm{n}=$ 32). The study period was 12 weeks. In group E, $10 \mathrm{mg}$ of ezetimibe was administered daily after breakfast. Serum lipids were measured every 4 weeks, while lathosterol (a cholesterol synthesis marker) and campesterol and sitosterol (cholesterol absorption markers) were examined at baseline and at 12 weeks.
\end{abstract}

Results: A significant reduction of LDL-C was observed in group E at both 4 and 12 weeks (from $155 \pm 3.9$ to $128 \pm 3.4 \mathrm{mg} / \mathrm{dL}$ and 132 $\pm 3.9 \mathrm{mg} / \mathrm{dL}$, respectively, both $\mathrm{P}<0.01$ ), associated with an increase of high-density lipoprotein cholesterol (HDL-C)at 12 weeks (from $53 \pm 1.3$ to $55 \pm 1.5 \mathrm{mg} / \mathrm{dL}, \mathrm{P}<0.05)$ and no change of triglycerides. In contrast, none of these lipids changed in group C. An increase of lathosterol and a decrease of campesterol and sitosterol were observed in group E, while none of these markers changed in group C. When group E was divided into two subgroups according to the reduction of LDL-C, which were a good response group (reduction $\geq$ $20 \mathrm{mg} / \mathrm{dL}, \Delta \mathrm{LDL}-\mathrm{C}=-27.9 \pm 1.3 \mathrm{mg} / \mathrm{dL}, \mathrm{n}=18$ ) and a poor response group (reduction $<20 \mathrm{mg} / \mathrm{dL}, \Delta \mathrm{LDL}-\mathrm{C}=-3.7 \pm 2.5 \mathrm{mg} / \mathrm{dL}, \mathrm{n}=26$ ), baseline levels of campesterol and sitosterol were higher in the good response group.

Conclusion: Ezetimibe monotherapy reduced LDL-C and increased

Manuscript accepted for publication October 19, 2016

aDepartment of Internal Medicine, Division of Metabolism and Endocrinology, St. Marianna University School of Medicine, 2-16-1, Sugao, Miyamaeku, Kawasaki, Kanagawa, 216-8511, Japan

${ }^{\mathrm{b}}$ Corresponding Author: Akio Ohta, Department of Internal Medicine, Division of Metabolism and Endocrinology, St. Marianna University School of Medicine, Toyoko Hospital, 3-435, Kosugi-tyou, Nakahara-ku, Kawasaki, Kanagawa 211-0063, Japan. Email: a2oota@marianna-u.ac.jp

doi: https://doi.org/10.14740/jocmr2782w
HDL-C, with the reduction of LDL-C being greater in patients with higher levels of cholesterol absorption markers.

Keywords: Ezetimibe; Monotherapy; Campesterol; Sitosterol; Lathosterol

\section{Introduction}

Elevation of serum cholesterol, especially low-density lipoprotein cholesterol (LDL-C), is a significant risk factor for cardiovascular disease (CVD), and the target level for serum LCL-C is established according to the patient's clinical profile [1-3]. The serum cholesterol level reflects intestinal absorption of cholesterol from the diet and bile as well as cholesterol production in the liver. Statins potently inhibit HMG-CoA reductase and thus reduce hepatic synthesis of cholesterol, so patients with elevation of LDL-C due to overproduction of cholesterol should be treated with statins, and these drugs are widely used as first-line therapy to reduce the serum LDL-C level and prevent CVD [46]. However, a satisfactory LCL-C level is not achieved by statin monotherapy in some patients, suggesting that these patients may need treatment with inhibitors of intestinal cholesterol absorption. Ezetimibe selectively inhibits the intestinal absorption of dietary and biliary cholesterol, and reduction of LDL-C and the risk of CAD by treatment with this drug have been reported $[7,8]$. The main action of ezetimibe is to block Niemann-Pick C1-like 1 transporter (NPC1L1), which has an essential role in the absorption and reabsorption of cholesterol and other plant sterols from the intestine and liver $[9,10]$.

Several biomarkers of cholesterol synthesis and absorption have been developed recently [11]. The intermediate metabolites squalene, lathosterol, and desmosterol are known as biomarkers of cholesterol synthesis, and there is a linear relationship between their serum concentrations and the activity of hepatic HMG-CoA reductase, which is the key enzyme in cholesterol synthesis. Since the correlation with HMG-CoA reductase activity is strongest for lathosterol [12], it is commonly used as a biomarker of cholesterol synthesis. As biomarkers of cholesterol absorption, the serum concentrations of two dietary plant sterols (campesterol and sitosterol) were measured. These substances are transported by incorporation into lipoproteins, mainly LDL as well as cholesterol. Both statins and ezetimibe reduce the serum level of LDL-C, and it 
has been reported that statins also reduce the cholesterol synthesis marker lathosterol, while ezetimibe decreases the absorption markers campesterol and sitosterol [11, 13]. However, the effect of ezetimibe on cholesterol synthesis and absorption markers has not been fully evaluated in Japanese patients, nor has the association between the extent of LDL-C reduction by ezetimibe and baseline levels of campesterol and sitosterol or changes of these markers during ezetimibe treatment. Accordingly, the aim of the present study was to assess the effect of ezetimibe on LDL-C and on these cholesterol markers, and to evaluate the associations among these parameters in Japanese patients with hypercholesterolemia.

\section{Methods}

\section{Patients}

A total of 76 Japanese patients with hypercholesterolemia who were not on statin therapy and were not overweight or obese were recruited from the Outpatient Clinic of St. Marianna University Hospital (Kawasaki, Japan). The inclusion criteria were persistent elevation of serum LDL-C to $\geq 120 \mathrm{mg} / \mathrm{dL}$ despite restriction of dietary cholesterol intake $(<300 \mathrm{mg} /$ day) for at least 6 months without lipid-lowering therapy and no diabetes (fasting plasma glucose $<140 \mathrm{mg} / \mathrm{dL}$ and $\mathrm{HbA} 1 \mathrm{c}<6.5 \%$ ). Exclusion criteria were pregnancy, severe illness, anemia, renal failure (serum creatinine $>2.0 \mathrm{mg} / \mathrm{dL}$ ), chronic liver disease, thyroid disease, and malignancy. All patients gave written informed consent to this study and it was approved by the Ethics Committee of St. Marianna University School of Medicine.

\section{Study design}

After enrollment, the patients were randomized to two groups, which were an ezetimibe group (group $\mathrm{E}, \mathrm{n}=44$ ) and a control group without ezetimibe that received diet therapy alone (group $\mathrm{C}, \mathrm{n}=32$ ). Group $\mathrm{E}$ received ezetimibe at $10 \mathrm{mg} /$ day after breakfast without any change of dosage throughout the study, and the daily total energy intake was set at $25 \mathrm{kcal} / \mathrm{kg}$ of ideal body weight. Group $\mathrm{C}$ did not take medications and ate a diet with the same energy intake and nutrient balance $(25 \%$ fat, $60 \%$ carbohydrates, and $15 \%$ protein), as well as the same dietary cholesterol intake $(<300 \mathrm{mg} /$ day $)$. Alcohol consumption was restricted to less than $20 \mathrm{~g} /$ day and daily exercise was set at $150-200 \mathrm{kcal} / \mathrm{day}$. The study period was 12 weeks and the patients visited the outpatient clinic every 4 weeks. Throughout the study, all patients were encouraged to continue their diet and exercise therapy by the attending doctor and other clinic staff.

Blood samples were obtained after an overnight fast. Serum triglycerides (TG), high-density lipoprotein cholesterol (HDL-C), LDL-C, and plasma glucose were measured by standard methods at the central study laboratory. The TG concentration was determined by an enzymatic method using an autoanalyzer (Determiner TG, Kyowa Medex Co., Ltd, Shizuoka, Japan). HDL-C and LDL-C were measured by the direct method (Kyowa Metabolead Standard Serum HDL/LDL-C,
Table 1. Baseline Characteristics of the Patients

\begin{tabular}{llll}
\hline & E group & C group & P-value \\
\hline Gender (male/female) & $44(18: 26)$ & $32(17: 15)$ & 0.35 \\
Age $($ years $)$ & $59.3 \pm 2.1$ & $62.6 \pm 2.2$ & 0.28 \\
BMI $\left(\mathrm{kg} / \mathrm{m}^{2}\right)$ & $28.1 \pm 1.1$ & $27.4 \pm 0.6$ & 0.59 \\
FPG $(\mathrm{mg} / \mathrm{dL})$ & $105 \pm 3$ & $108 \pm 4$ & 0.53 \\
HbA1c $(\%)$ & $5.5 \pm 0.1$ & $5.6 \pm 0.1$ & 0.68 \\
AST $(\mathrm{mg} / \mathrm{dL})$ & $24.6 \pm 1.5$ & $22.3 \pm 1.1$ & 0.25 \\
ALT $(\mathrm{mg} / \mathrm{dL})$ & $27.8 \pm 3.4$ & $24.8 \pm 2.8$ & 0.53 \\
Creatinine $(\mathrm{mg} / \mathrm{dL})$ & $0.7 \pm 0.0$ & $0.7 \pm 0.0$ & 0.58 \\
TG $(\mathrm{mg} / \mathrm{dL})$ & $150.7 \pm 10.8$ & $141.3 \pm 11.2$ & 0.55 \\
HDL-C $(\mathrm{mg} / \mathrm{dL})$ & $52.8 \pm 1.3$ & $54.1 \pm 2.3$ & 0.60 \\
LDL-C $(\mathrm{mg} / \mathrm{dL})$ & $154.6 \pm 3.9$ & $152.5 \pm 3.7$ & 0.71 \\
RLP-C $(\mathrm{mg} / \mathrm{dL})$ & $7.3 \pm 0.6$ & $6.4 \pm 0.5$ & 0.28 \\
Lathosterol $(\mu \mathrm{g} / \mathrm{mL})$ & $4.2 \pm 0.3$ & $4.2 \pm 0.3$ & 0.95 \\
Campesterol $(\mu \mathrm{g} / \mathrm{mL})$ & $5.7 \pm 0.4$ & $5.6 \pm 0.4$ & 0.88 \\
Sitosterol $(\mu \mathrm{g} / \mathrm{mL})$ & $3.2 \pm 0.2$ & $3.1 \pm 0.2$ & 0.65 \\
\hline
\end{tabular}

Results are expressed as the mean \pm SE. E group: ezetimibe treated group. C group: ezetimibe non-treated control group. BMI: body mass index; FPG: fasting plasma glucose; HbA1c: hemoglobin A1c; GA: glycated albumin; HDL-C: high-density lipoprotein cholesterol; LDL-C: low-density lipoprotein cholesterol; TG: triglycerides; RLP-C: remnantlike particles cholesterol.

Kyowa Medex Co., Ltd, Shizuoka, Japan). Serum concentrations of the markers of cholesterol synthesis (lathosterol) and absorption (campesterol and sitosterol) were measured by gas chromatography, while serum insulin was measured by chemiluminescent immunoassay (CLIA) using a commercial kit (Architect Insulin; Abbott Japan Co., Ltd, Tokyo, Japan). Homeostasis model assessment of insulin resistance (HOMA-IR) was performed as described previously $[13,14]$. HbA1c was determined by the latex cohesion method (Determiner HbA1c, Kyowa Medex, Tokyo, Japan).

\section{Statistical analysis}

Results are expressed as the mean \pm SE. Differences among variables measured at baseline, 4 weeks, and 12 weeks were determined by one-way ANOVA. The two-tailed unpaired $t$ test was used for comparisons between two groups. All analyses were performed with Stat-View software (Abacus Concepts, Berkeley, CA). A probability value of less than 0.05 was considered to indicate statistical significance.

\section{Results}

\section{Baseline characteristics and lipid profile of the patients}

As shown in Table 1, baseline characteristics of the patients did not differ between the two groups. With regard to glucose 

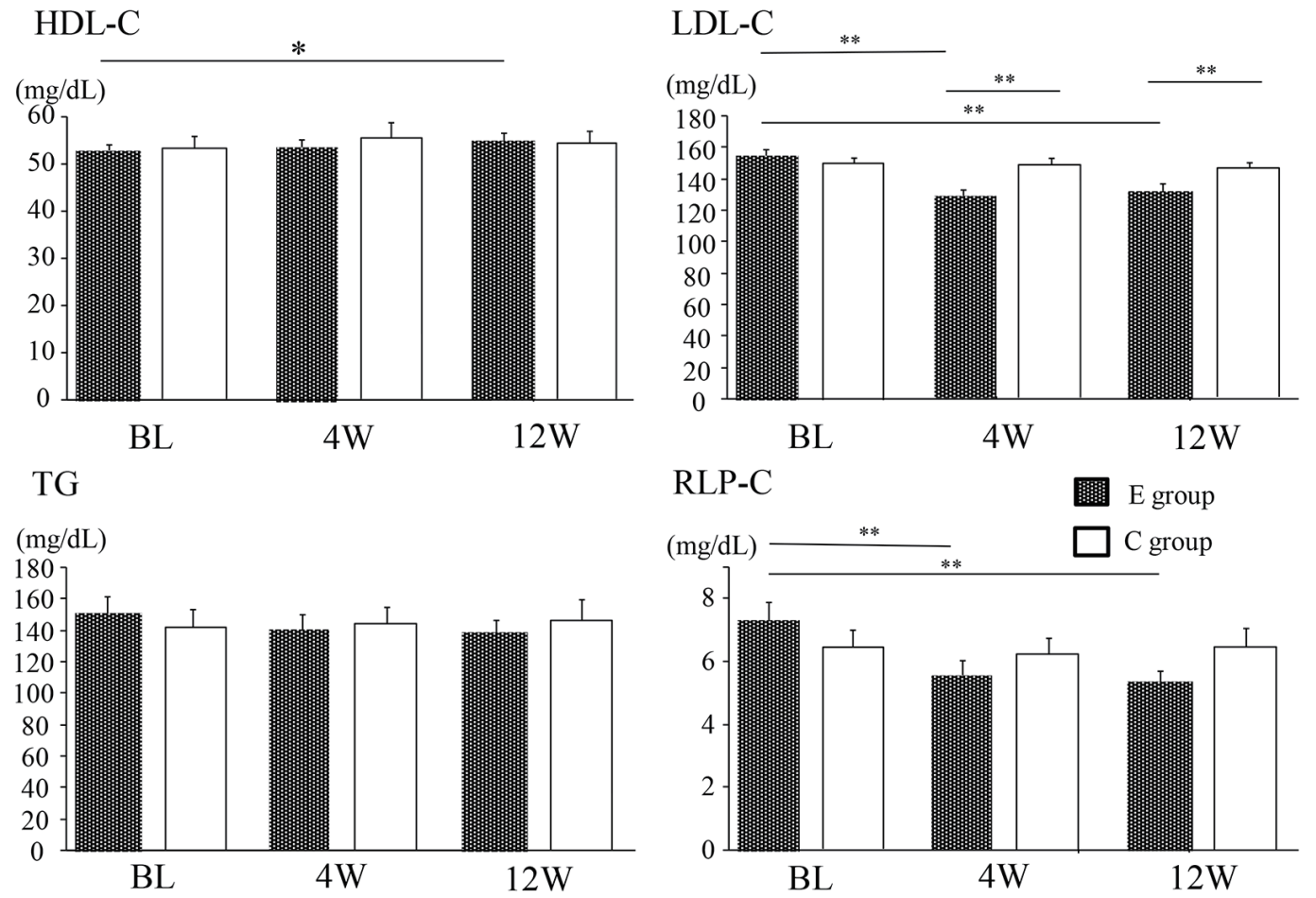

Figure 1. Changes of the lipid profile in the $E$ group and $C$ group. Data are shown as the mean $\pm S E$. BL: baseline. ${ }^{*} P<0.05$, ${ }^{*} P$ $<0.01$. E group: ezetimibe treated group. C group: ezetimibe non-treated control group.

metabolism, there were no significant changes of body mass index (BMI), fasting plasma glucose, HbAlc, and HOMA-IR throughout the study period in both groups. Figure 1 displays serum lipids at baseline, 4 weeks, and 12 weeks. In group E, LDL-C showed a significant decrease from the baseline level at 4 and 12 weeks (from $155 \pm 3.9$ to $128 \pm 3.4 \mathrm{mg} / \mathrm{dL}$ and 132 $\pm 3.9 \mathrm{mg} / \mathrm{dL}$, respectively, both $\mathrm{P}<0.01$ ), while HDL-C was significantly increased from baseline at 12 weeks (from $53 \pm$ 1.3 to $55 \pm 1.5 \mathrm{mg} / \mathrm{dL}, \mathrm{P}<0.05)$. A significant reduction of remnant-like particles cholesterol (RLP-C) from baseline was also observed in group $\mathrm{E}$ at 4 and 12 weeks (from $7.3 \pm 0.6$ to $5.5 \pm 0.5 \mathrm{mg} / \mathrm{dL}$ and $5.3 \pm 0.3 \mathrm{mg} / \mathrm{dL}$, respectively, both $\mathrm{P}<$ $0.01)$. In contrast, there were no changes of LDL-C, HDL-C, and RLP-C in group C (LDL-C: from $153 \pm 3.7$ to $150 \pm 4.3$ and $147 \pm 3.2 \mathrm{mg} / \mathrm{dL}$, HDL-C: from $54 \pm 2.3$ to $55 \pm 2.7 \mathrm{mg} /$ $\mathrm{dL}, \mathrm{RLP}-\mathrm{C}$ : from $6.4 \pm 0.5$ to $6.3 \pm 0.5$ and $6.5 \pm 0.6 \mathrm{mg} / \mathrm{dL})$. TG showed no significant changes at 4 and 12 weeks in groups (group E: from $151 \pm 11$ to $139 \pm 10$ and $139 \pm 7.4 \mathrm{mg} / \mathrm{dL}$, group C: from $141 \pm 11$ to $144 \pm 10$ and $146 \pm 13 \mathrm{mg} / \mathrm{dL}$ ).

\section{Changes of cholesterol absorption and synthesis markers}

Table 2 displays the levels of serum lipids, the cholesterol synthesis marker (lathosterol), and the cholesterol absorption markers (campesterol and sitosterol) at baseline and 12 weeks. An increase of lathosterol and a decline of both campesterol and sitosterol were observed in group $\mathrm{E}$, but none of

Table 2. Comparison of the Changes in Serum Lipids and Cholesterol Absorption and Synthesis Markers

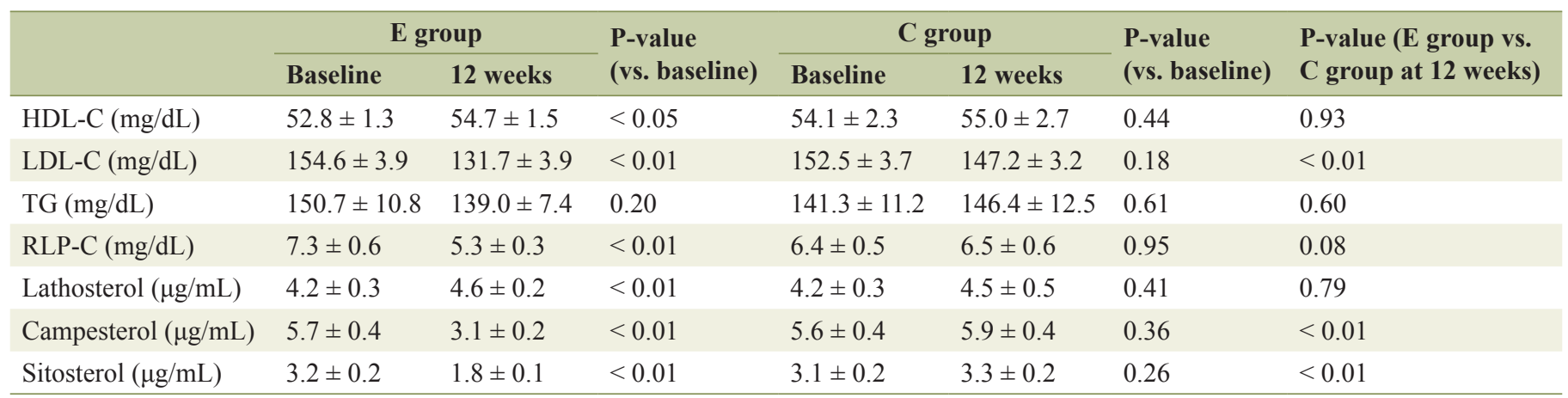

Results are expressed as the mean \pm SE. E group: ezetimibe treated group. C group: ezetimibe non-treated control group. 

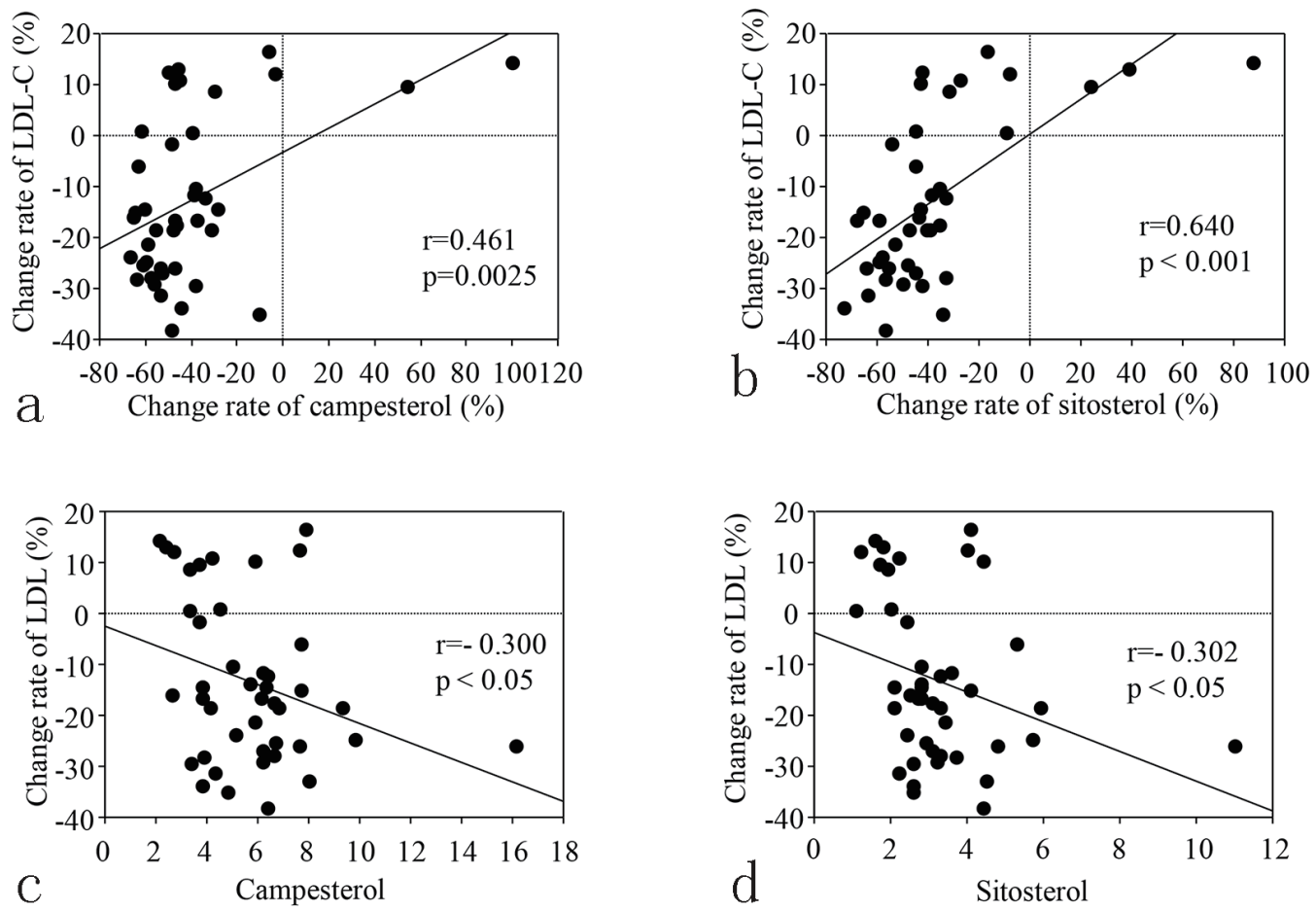

Figure 2. $(a, b)$ Correlation between changing rate of LDL-C and changing rate of cholesterol absorption markers from baseline to 12 weeks in the E group. (c, d) Correlation between changing rate of LDL-C from baseline to 12 weeks and the baseline levels of cholesterol absorption markers in the $\mathrm{E}$ group.

these markers changed in group $\mathrm{C}$. The correlation between the percent change of LDL-C from baseline to 12 weeks and the change of campesterol or sitosterol during the same period is shown in Figure $2 \mathrm{a}$ and $\mathrm{b}$, respectively, while correlations between the percent change of LDL-C and the baseline levels of these markers are shown in Figure $2 \mathrm{c}$ and $\mathrm{d}$. The percent change of LDL-C was positively correlated with that of each marker, and was negatively correlated with the baseline level of each marker.

\section{Ezetimibe responders}

Group E was divided into the two subgroups according to the extent of LDL-C reduction, which were a good response group $(\geq 20 \mathrm{mg} / \mathrm{dL}$ reduction, $\mathrm{n}=18)$ and a poor response group $(<$ $20 \mathrm{mg} / \mathrm{dL}$ reduction, $\mathrm{n}=26$ ). The good response group showed greater reduction of LDL-C than the poor response group $(-27.9 \pm 1.3$ vs. $-3.7 \pm 2.5 \mathrm{mg} / \mathrm{dL}, \mathrm{P}<0.01)$. A comparison of baseline characteristics, lipids, and biomarkers between these two groups is shown in the Table 3 . Baseline levels of campesterol and sitosterol were higher in the good response group than in the poor response group, but the other variables did not differ between the two groups.

\section{Discussion}

There were three main findings of the present study. First, 12 weeks of ezetimibe monotherapy reduced the serum level of LDL-C and increased HDL-C, but did not alter TG.

Second, cholesterol absorption markers (campesterol and sitosterol) were decreased by ezetimibe treatment, while a cholesterol synthesis marker (lathosterol) was increased. According to previous reports, ezetimibe decreases the levels of the cholesterol absorption markers campesterol and sitosterol by $20-50 \%$, while increasing the cholesterol synthesis marker lathosterol by $0-80 \%[11,13]$, suggesting that compensatory upregulation of hepatic cholesterol synthesis occurs after suppression of intestinal cholesterol absorption. In contrast, statin therapy decreases lathosterol by $40-70 \%$, while campesterol is increased by $0-70 \%$ and sitosterol by $0-90 \%[11,13]$, suggesting compensatory upregulation of intestinal cholesterol absorption in patients taking statins. Consistent with these reports, the present study showed that campesterol was decreased by $40.6 \pm 4.9 \%$ and sitosterol was reduced by $38.1 \pm 4.7 \%$ after 12 weeks of ezetimibe monotherapy, along with elevation of lathosterol by $24.5 \pm 5.3 \%$. Since the cholesterol-lowering effect of ezetimibe depends on inhibiting intestinal cholesterol absorption, the two cholesterol absorption markers would be expected to change in parallel with LDL-C.

Third, the percent reduction of LDL-C by ezetimibe was positively correlated with the percent reduction of the cholesterol absorption markers (campesterol and sitosterol) and negatively correlated with the baseline levels of these markers. That is, the extent of the decrease in LDL-C became larger along with stronger suppression of the two cholesterol absorption markers by ezetimibe, as shown in Figure 2. 
Table 3. Comparison of Baseline Parameters Between the Ezetimibe High Responder Group and the Low Responder Group

\begin{tabular}{llll}
\hline & High responder group & Low responder group & P-value \\
\hline $\mathrm{n}$ & 18 & 26 & \\
Age $($ years $)$ & $61.8 \pm 2.7$ & $57.8 \pm 2.9$ & 0.36 \\
BMI $\left(\mathrm{kg} / \mathrm{m}^{2}\right)$ & $27.0 \pm 1.9$ & $28.1 \pm 1.5$ & 0.41 \\
FPG $(\mathrm{mg} / \mathrm{dL})$ & $102 \pm 5$ & $106 \pm 4$ & 0.49 \\
HDL-C $(\mathrm{mg} / \mathrm{dL})$ & $53.4 \pm 2.0$ & $52.3 \pm 1.7$ & 0.69 \\
LDL-C $(\mathrm{mg} / \mathrm{dL})$ & $163.1 \pm 4.0$ & $148.7 \pm 5.7$ & 0.07 \\
RLP-C $(\mathrm{mg} / \mathrm{dL})$ & $6.8 \pm 0.8$ & $7.6 \pm 0.8$ & 0.49 \\
Lathosterol $(\mu \mathrm{g} / \mathrm{mL})$ & $4.2 \pm 0.3$ & $4.2 \pm 0.4$ & 0.98 \\
Campesterol $(\mu \mathrm{g} / \mathrm{mL})$ & $6.6 \pm 0.7$ & $5.1 \pm 0.4$ & 0.04 \\
Sitosterol $(\mu \mathrm{g} / \mathrm{mL})$ & $3.8 \pm 0.5$ & $2.8 \pm 0.2$ & 0.04 \\
\hline
\end{tabular}

Results are expressed as the mean $\pm \mathrm{SE}$. High responder group: equal or more than $20 \mathrm{mg} / \mathrm{dL}$ LDL-C reduction. Low responder group: less than $20 \mathrm{mg} / \mathrm{dL}$ LDL-C reduction.

Also, the decrease of LDL-C was greater in patients with higher baseline levels of the two cholesterol absorption markers. These results reflect the pharmacologic mechanism of ezetimibe, which means that its efficacy is related to the extent of inhibition of cholesterol absorption.

Furthermore, baseline levels of the cholesterol absorption markers were higher in the patients with a good response to ezetimibe ( $\geq 20 \mathrm{mg} / \mathrm{dL}$ reduction of LDL-C) than in patients with a poor response, despite no significant difference of the baseline LDL-C level (Table 3). Taken together, these findings suggest that greater reduction of LDL-C by ezetimibe can be expected in patients with higher baseline levels of the two cholesterol absorption markers.

Strict control of LDL-C is necessary to prevent CVD, and combined administration of ezetimibe with a statin is recommended if the LDL-C level remains unsatisfactory in patients on statin monotherapy $[14,15]$. Recently, Katsura et al reported the effect of ezetimibe treatment for 12 weeks on LDL-C in Japanese patients with type 2 diabetes (LDL-C $\geq 120 \mathrm{mg}$ / $\mathrm{dL}$ or $\geq 100 \mathrm{mg} / \mathrm{dL}$ with ischemic heart disease) with or without concomitant statin therapy [16], and they demonstrated a larger percent reduction of LDL-C in the patients using statins than in those without statins $(-31.4 \%$ vs. $-18.4 \%)$. The reduction of LDL-C achieved with ezetimibe monotherapy in the present study $(-13.3 \pm 2.4 \%)$ was similar to that reported by Katsura and by other authors [17]. Interestingly, Katsura et al also examined lathosterol, campesterol, and sitosterol at baseline and 12 weeks, and found higher baseline levels of campesterol and sitosterol with a lower level of lathosterol in the statin group compared with the non-statin group. Regarding this point, they mentioned that ezetimibe may have a stronger effect in patients with higher baseline levels of cholesterol absorption markers, which in turn may lead to greater reduction of LDL-C. Their data were consistent with our findings in the present study, and both studies suggest that ezetimibe may be useful when combined with statin therapy to achieve the target LDL-C level for prevention of atherosclerosis and CVD. Actually, the combination of statin therapy and ezetimibe has been reported to achieve greater coronary plaque regression than statin monotherapy in Japanese patients [18].

The present study had several limitations. First, the number of subjects was small and the study period was relatively short. Long-term evaluation in a large study population would be needed to confirm the usefulness of ezetimibe for Japanese patients. Second, we did not compare the effect of ezetimibe monotherapy with that of ezetimibe/statin combination therapy. As discussed above, greater reduction of LDL-C can be expected with combination therapy, so performing such a study could provide more information on the clinical efficiency of ezetimibe in Japanese patients. Third, we did not assess NPC1L1 gene polymorphism. Recent studies have indicated that several SNPs of NPC1L1 may have an influence on cholesterol absorption $[19,20]$. To further investigate individual differences of the baseline sitosterol and campesterol levels and identify indicators of the response to ezetimibe, a future study with strict control of dietary cholesterol intake and SNP analysis of NPC1L1 may be needed.

In conclusion, ezetimibe monotherapy reduced LDL$\mathrm{C}$ levels along with a decrease of sitosterol and campesterol (cholesterol absorption markers) and an increase of lathosterol (cholesterol synthesis marker) in Japanese patients with hypercholesterolemia. The effect of ezetimibe was greater in patients with higher baseline sitosterol and campesterol levels than in patients with lower levels of the markers.

\section{Acknowledgments}

The authors thank all of the investigators and patients involved in this study.

\section{Conflicts of Interest}

The authors have no conflicts of interest that are relevant to the content of this manuscript. 


\section{Funding}

No sources of funding were used to assist in the preparation of this manuscript.

\section{References}

1. Hayward RA, Krumholz HM, Zulman DM, Timbie JW, Vijan S. Optimizing statin treatment for primary prevention of coronary artery disease. Ann Intern Med. 2010;152(2):69-77.

2. Saposnik G, Fonarow GC, Pan W, Liang L, Hernandez AF, Schwamm LH, Smith EE. Guideline-directed lowdensity lipoprotein management in high-risk patients with ischemic stroke: findings from Get with the GuidelinesStroke 2003 to 2012. Stroke. 2014;45(11):3343-3351.

3. Naito R, Miyauchi K, Konishi H, Tsuboi S, Ogita M, Dohi T, Kasai T, et al. Appropriate Level of Low-Density Lipoprotein Cholesterol for Secondary Prevention of Coronary Artery Disease. J Atheroscler Thromb. 2016;23(4):413-421.

4. Pursnani A, Massaro JM, D'Agostino RB, Sr., O'Donnell CJ, Hoffmann U. Guideline-Based Statin Eligibility, Coronary Artery Calcification, and Cardiovascular Events. JAMA. 2015;314(2):134-141.

5. Lee JC, Zdrojewski T, Pencina MJ, Wyszomirski A, Lachacz M, Opolski G, Bandosz P, et al. Population Effect of Differences in Cholesterol Guidelines in Eastern Europe and the United States. JAMA Cardiol. 2016;1(6):700-707.

6. Teramoto T, Uno K, Miyoshi I, Khan I, Gorcyca K, Sanchez RJ, Yoshida S, et al. Low-density lipoprotein cholesterol levels and lipid-modifying therapy prescription patterns in the real world: An analysis of more than 33,000 high cardiovascular risk patients in Japan. Atherosclerosis. 2016;251:248-254.

7. Battaggia A, Donzelli A, Font M, Molteni D, Galvano A. Clinical efficacy and safety of Ezetimibe on major cardiovascular endpoints: systematic review and metaanalysis of randomized controlled trials. PLoS One. 2015;10(4):e0124587.

8. Knopp RH, Dujovne CA, Le Beaut A, Lipka LJ, Suresh R, Veltri EP. Evaluation of the efficacy, safety, and tolerability of ezetimibe in primary hypercholesterolaemia: a pooled analysis from two controlled phase III clinical studies. Int J Clin Pract. 2003;57(5):363-368.

9. Altmann SW, Davis HR, Jr., Zhu LJ, Yao X, Hoos LM, Tetzloff G, Iyer SP, et al. Niemann-Pick C1 Like 1 protein is critical for intestinal cholesterol absorption. Science. 2004;303(5661):1201-1204.

10. Johnson TA, Pfeffer SR. Ezetimibe-sensitive cholesterol uptake by NPC1L1 protein does not require endocytosis. Mol Biol Cell. 2016;27(11):1845-1852.

11. Wu AH. Biomarkers for cholesterol absorption and synthesis in hyperlipidemic patients: role for therapeutic selection. Clin Lab Med. 2014;34(1):157-166, viii.

12. Bjorkhem I, Miettinen T, Reihner E, Ewerth S, Angelin B, Einarsson K. Correlation between serum levels of some cholesterol precursors and activity of HMG-CoA reductase in human liver. J Lipid Res. 1987;28(10):1137-1143.

13. Descamps OS, De Sutter J, Guillaume M, Missault L. Where does the interplay between cholesterol absorption and synthesis in the context of statin and/or ezetimibe treatment stand today? Atherosclerosis. 2011;217(2):308321.

14. Sakamoto K, Kawamura M, Kohro T, Omura M, Watanabe T, Ashidate K, Horiuchi T, et al. Effect of Ezetimibe on LDL-C Lowering and Atherogenic Lipoprotein Profiles in Type 2 Diabetic Patients Poorly Controlled by Statins. PLoS One. 2015;10(9):e0138332.

15. Tsujita K, Yamanaga K, Komura N, Sakamoto K, Sugiyama S, Sumida H, Shimomura H, et al. Synergistic effect of ezetimibe addition on coronary atheroma regression in patients with prior statin therapy: Subanalysis of PRECISE-IVUS trial. Eur J Prev Cardiol. 2016;23(14):15241528.

16. Katsura T, Katakami N, Irie Y, Yamamoto Y, Okusu T, Kubo F, Kanamaru Y, et al. The usefulness of a cholesterol absorption inhibitor in Japanese type 2 diabetes patients with dyslipidemia. Diabetes Technol Ther. 2015;17(6):427-434.

17. Hiramitsu S, Ishiguro $Y$, Matsuyama H, Yamada K, Kato $\mathrm{K}$, Noba M, Uemura A, et al. The effects of ezetimibe on surrogate markers of cholesterol absorption and synthesis in Japanese patients with dyslipidemia. J Atheroscler Thromb. 2010;17(1):106-114.

18. Tsujita K, Sugiyama S, Sumida H, Shimomura H, Yamashita T, Yamanaga K, Komura N, et al. Impact of Dual Lipid-Lowering Strategy With Ezetimibe and Atorvastatin on Coronary Plaque Regression in Patients With Percutaneous Coronary Intervention: The Multicenter Randomized Controlled PRECISE-IVUS Trial. J Am Coll Cardiol. 2015;66(5):495-507.

19. Lupattelli G, Pisciotta L, De Vuono S, Siepi D, Bellocchio A, Melis F, Bertolini S, et al. A silent mutation of Niemann-Pick C1-like 1 and apolipoprotein E4 modulate cholesterol absorption in primary hyperlipidemias. J Clin Lipidol. 2013;7(2):147-152.

20. Kashiwabara Y, Kobayashi Y, Koba S, Kohyama N, Ohbayashi M, Murayama JI, Hirano T, et al. Gene polymorphism and frequencies of the NPC1L1 gene (rs2072183, rs217434 and rs217428) in Japanese patients with dyslipidemia. J Clin Pharm Ther. 2014;39(5):551-554. 